\title{
THOSE UBIQUITOUS, BUT OFT UNSTABLE, LATTICE SOLITONS ${ }^{\dagger}$
}

\author{
C. F. DRISCOLL AND T. M. O'NEIL
}

\begin{abstract}
The soliton is the dominant characteristic of one-dimensional, nonlinear lattices. It is shown that these lattice solitons commonly manifest exponential instabilities. The modified KortewegdeVries equation is shown to have unstable solutions which quantitatively model the instability on the cubicly nonlinear lattice. In contrast, the quadratically nonlinear idttice shows an instability which scales with terms neglected in the $K d V$ approximation.
\end{abstract}

Introduction. The study of nonlinear dynamical systems has been motivated by the desire to understand effects which have traditionally been treated statistically. The justification of the ergodic hypothesis of thermodynamics and the finite heat conductivity of nonconducting crystals are two such problems. Fermi, Pasta, and Ulam [1] considered the one-dimensional anharmonic lattice as a simple model which was expected to show ergodic behavior and thermalization. The observation of periodic recurrences and the apparent lack of randomization were startling results which required explanation.

This led Zabusky and Kruskal [2] to the discovery that the lattice dynamics is dominated by solitary propagating pulses, or solitons; the observed modal recurrences are seen to be echoes from essentially freestreaming solitons. The analysis was facilitated by relating the discrete lattice to the continuum Korteweg-deVries $(K d V)$ equation. With the development of analytic methods to solve nonlinear equations, and the numerical simulation of other physical systems, the soliton has been established as the fundamental mode of many nonlinear systems [3].

The concept of the stable, free-streaming soliton has seemed at odds, however, with the original desire to understand randomization and equipartition of energy. The continuing work on lattice wave randomization has focused on exponential instabilities as the basis for stochastic effects: an infinitesimal perturbation in initial conditions will grow exponentially and completely alter the dynamics. This work on lattice instabilities [4], [5], [6] has been largely independent of the work on solitons.

In this paper, we take a first step toward relating lattice instabilities to solitons and the $K d V$ approximation. We find that lattice solitons are

†Work supported by National Science Foundation Grant PHY-73-05125-A02 and A. P. Sloan Grant BR-1323. 
not as perfectly stable as often supposed, but rather commonly exhibit exponential instabilities. The quadratically nonlinear lattice shows an instability which scales to zero in the continuum limit, corresponding to the known stability of $K d V$ solutions. The cubicly nonlinear lattice shows an instability which does not scale to zero in the continuum limit: analysis of the modified Korteweg-deVries $(m K d V)$ equation shows that some solutions are exponentially unstable, and the $m K d V$ instabilities agree quantitatively with the observed lattice instabilities. The lattice instabilities are seen to allow energy to flow into otherwise inaccessible modes, and may form the basis for at least partial randomization.

Nonlinear Lattice and $K d V$ Equations. Consider the one-dimensional nonlinear lattice governed by the dynamical equation

$$
\begin{aligned}
\frac{\partial^{2}}{\partial t^{2}} y_{j}= & \left(y_{j+1}-2 y_{j}+y_{j-1}\right) \\
& +\alpha\left[\left(y_{j+1}-y_{j}\right)^{p+1}-\left(y_{j}-y_{j-1}\right)^{p+1}\right] .
\end{aligned}
$$

Here, $y_{j}(t)$ is the displacement from equilibrium of the $j^{\text {th }}$ mass, $j=1$, $2 \cdots N$, and periodic boundary conditions are specified by $y_{0} \equiv y_{N}$ and $y_{N+1} \equiv y_{1}$. The nonlinear forcing term has coefficient $\alpha$ and exponent $p$; we sill consider the cases $p=1$ and $p=2$, that is, quadratic and cubic nonlinearities. The excitation of the nonlinear lattice may be viewed as a sum of linear Fourier modes,

$$
\begin{aligned}
y_{j}(t)= & \sum_{m} A_{m}(t) \exp \left(i \frac{2 \pi}{N} m j-i \omega_{m} t\right) \\
& +B_{m}(t) \exp \left(i \frac{2 \pi}{N} m j+i \omega_{m} t\right),
\end{aligned}
$$

with linear frequencies

$$
\omega_{m}=2 \sin (\pi m / N), \quad-N / 2 \leqq m \leqq N / 2 .
$$

The time evolution of the individual Fourier modes will in general be quite complicated, since the nonlinearity couples many modes together.

Zabusky and Kruskal [2] made two incisive approximations in order to understand the nonlinear evolution of lattice waves. First, the lattice displacement is considered to be a continuous function $y(x, t)$, with $y(j h, t)=y_{j}(t)$; here, $h \equiv L / N$, and $L$ is the system length, nominally 1 . Second, the two directions of wave propagation are considered separately. Waves travelling in the positive direction are represented by the variable 
(4) $u(x, t) \equiv \frac{-y_{t}}{2 h}+\frac{1}{2} \int_{0}^{\nu_{x}}\left[1+(p+1) \alpha h^{p} \eta^{p}\right]^{1 / 2} d \eta$,

where the subscripts $x$ and $t$ denote partial differentiation. The discrete differences in (1) are expressed as Taylor expansions, and only lowest order terms in nonlinearity and dispersion are kept. The neglected higher order terms are not important for soliton formation, but will be seen to be important in stability considerations. Then, by ignoring coupling to waves travelling in the negative direction, one obtains the equation

$$
u_{\tau}+\beta u^{p} u_{\xi}+\delta^{2} u_{\xi \xi \xi}=0 .
$$

For $p=1$ this is the $K d V$ equation, and for $p=2$ the $m K d V$ equation. Here, $\xi$ is the wave-frame spatial variable, $\xi \equiv x-h t$, and $\tau$ is a scaled time. The coefficients $\beta$ and $\delta^{2}$ may be scaled arbitrarily; in this paper we use

$$
\begin{array}{lll}
p=1: \beta \equiv 6, & \delta^{2}=h / 4 \alpha \equiv 1, & \tau=t h^{3} / 24, \\
p=2: \beta \equiv 12 \operatorname{sign}(\alpha), & \delta^{2}=1 / 3|\alpha| \equiv 1, & \tau=t h^{3} / 24 .
\end{array}
$$

For $p=1$, the sign of $\alpha$ may be reversed by the transformation $y \rightarrow(-y)$ in (1), so we consider only $\alpha>0$. For $p=2$, however, $\alpha>0$ and $\alpha<0$ are two distinct dynamical possibilities which result in the two possible signs for $\beta$. The magnitude of $\alpha$ is always chosen so as to make the $K d V$ or $m K d V$ parameter $\delta^{2}=1$; this amounts to a scaling of the magnitude $A$ of the lattice excitation, since from (1) the dynamics depends only on the product $\alpha A^{p}$.

The $K d V$ and $m K d V$ equations can be seen to have solitary propagating pulse solutions, or solitons. For example, the solutions

$$
\begin{aligned}
u(\xi, \tau) & =A \operatorname{sech}^{2 / p}[(\xi-b \tau) / \Delta], \\
\Delta & =\left(2 / A^{p} p^{2}\right)^{1 / 2}, \mathscr{b}=2 A^{p},
\end{aligned}
$$

propagate without change of form, for $\beta>0$ and $-\infty \leqq \xi \leqq \infty$. Furthermore, it is seen that general initial conditions form series of solitons, which maintain their individual identities even after passing through one another; that is, the solitons are persistent. It is this property which explains the behavior seen on the periodic lattice: the observed modal recurrences are echoes from essentially free-streaming solitons. The persistence of the individual solitons prevents complete randomization of modal energies. 
Lattice Instabilities. The fact that lattice solitons are persistent does not imply, however, that they are stable, in the usual sense of the word. Indeed, exponential instabilities are observed in lattice waves for both quadratic and cubic nonlinearities.

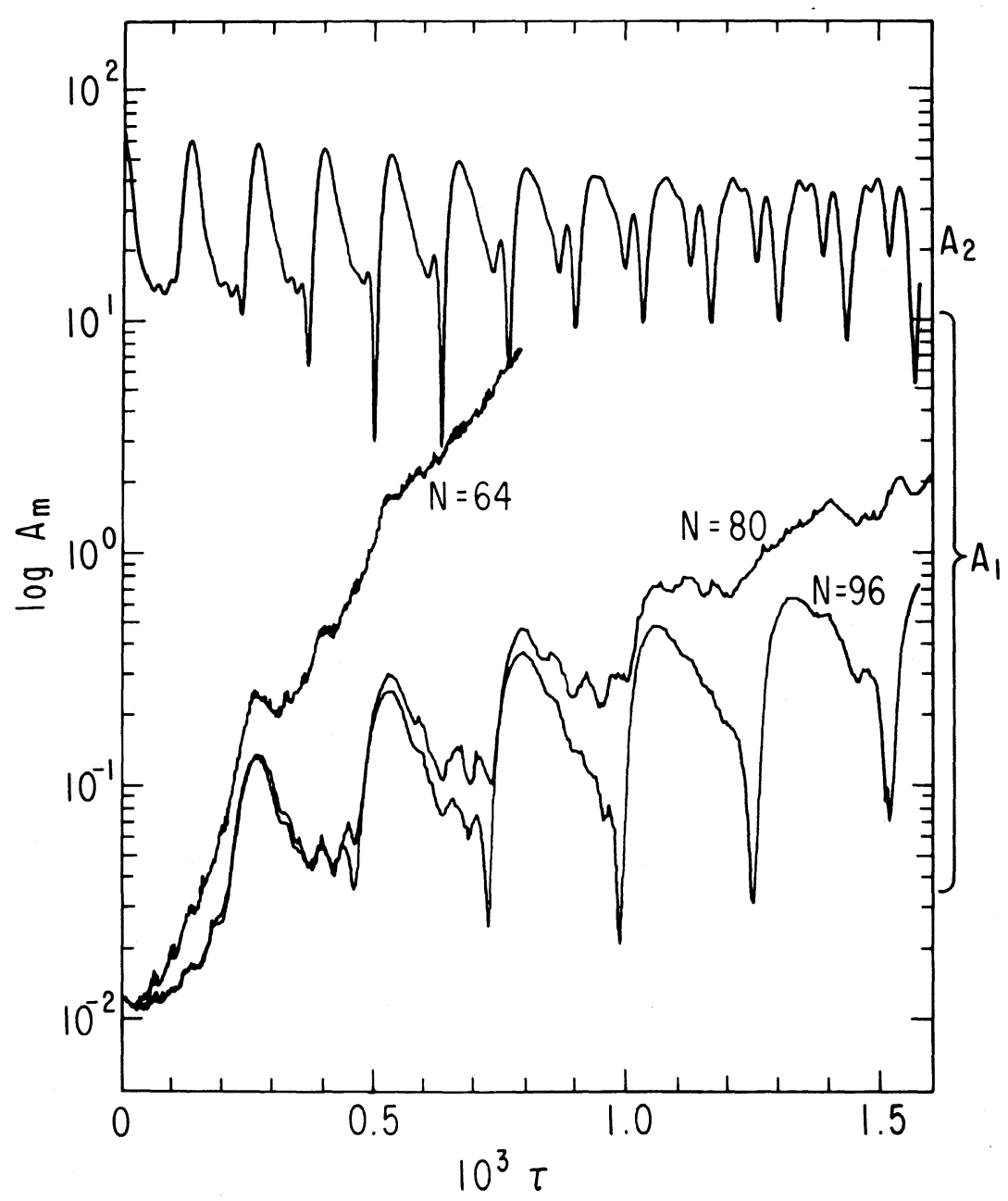

Figure 1. Lattice Fourier amplitudes $A_{1}$ and $A_{2}$ vs scaled time $\tau$ for three separate lattice integrations. The "recurrences" of mode 2 are the same for all three integrations, but the growth of mode 1 depends on the number of masses $N$. 
Consider first the quadratically nonlinear lattice, i.e., (1) with $p=1$. When a long wavelength Fourier mode, $A_{m}$, is excited with large amplitude, it is observed to couple nonlinearly to its harmonics; this corresponds to spatial steppening of the wave. As the wavefront becomes steeper, dispersive effects become important, and the wave breaks up into a series of individual solitons. These solitons stream with their individual velocities, and after lapping each other, return to near their original spatial positions. This causes the temporal recurrences of the original Fourier mode, as in the upper curve of Figure 1.

If the large amplitude mode is not the longest wavelength in the system, the possibility exists for decay of energy to longer wavelength, lower frequency modes. In Figure 1, we have excited mode 2 $\left(A_{2}=B_{2}=64\right)$, and observe growth from "noise" in mode 1 . Three separate lattice integrations are shown, each having a different number of masses $N$, but all having the same $K d V$ parameters $\beta$ and $\delta^{2}$. All three integrations show the same soliton formation, and the same recurrences of mode 2, on the $\tau$ time scale.

The growth of mode 1 is seen, however, to depend on $N$. In the continuum limit, only linear growth is seen: the peaks of the $N=96$ curve are equally spaced in time and have relative amplitudes $1.0,1.9,2.8$, $3.7,4.8$, and 5.8. The presence of mode 1 disturbs the symmetry of mode 2, making one group of solitons larger than the other. The difference in amplitudes causes a proportional difference in velocities. Every second recurrence, when the two groups are again distinct, the velocity differences result in a positional shift proportional to time. When Fourier analyzed, this appears as linear growth of mode 1 . As discreteness effects are increased, however, exponential growth is seen. For $N=80$, the exponential growth is comparable to the linear streaming, on the time scale of observation. When $N$ is decreased further to 64 , mode 1 is seen to grow in a clearly exponential manner. The discrete lattice thus manifests an exponential decay instability of variable strength.

The exponential growth of mode 1 may be seen to depend on the initial amplitude $A_{2}(0)$ as well as on $N$. We characterize the growth by its scaled exponentiation time $\tau_{e}$, or equivalently by the scaled growth rate $\gamma \equiv 1 / \tau_{e}$. Figure 2 displays $\gamma$ vs $h A_{2}$ (recall $\left.h=1 / N\right)$. We consider two types of initial conditions: "travelling waves" where only the positively travelling mode $A_{2}$ is excited; and "standing waves" where $B_{2}=A_{2}$, equivalent to fixed boundary conditions with half as many particles.

The dotted lines of Figure 2 show $\gamma \propto\left(\bar{h} A_{2}\right)^{3}$. This may also be written $\gamma \propto\left(h / \Delta^{2}\right)^{3} \propto\left(h^{2} / \Delta^{2}\right)^{1.5} \Delta^{-3}$, using the soliton spatial scale $\Delta$ of $(7)$. The ratio $h^{2} / \Delta^{2}$ is the basic expansion parameter of the $K d V$ approx- 


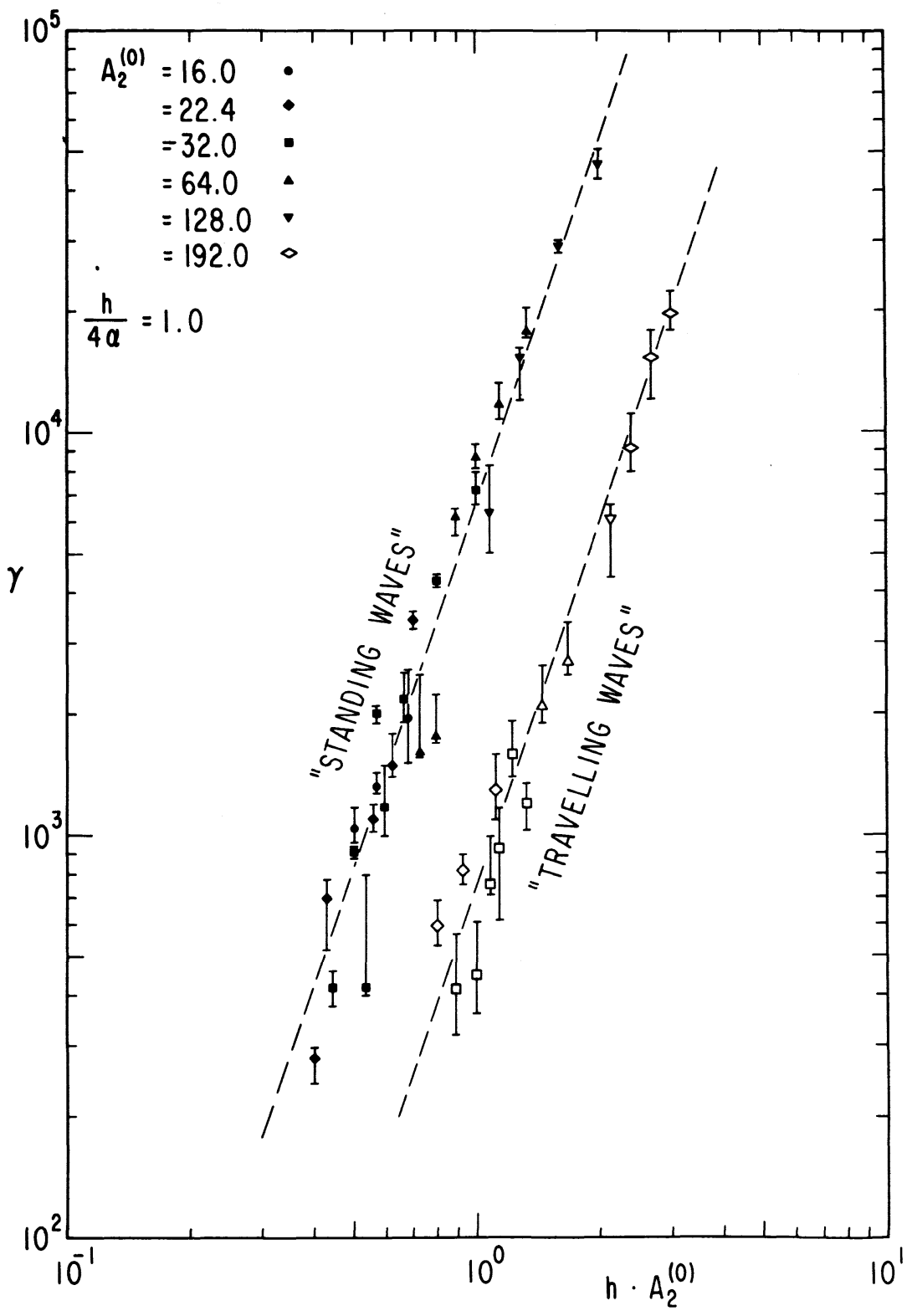

Figure 2. Scaled instability rate $\gamma$ vs "discreteness parameter" $h A_{2}(0)$, for the quadratically nonlinear lattice. 
imation, and gives the relative magnitude of the terms neglected in obtaining the $K d V$ equation. In the continuum limit of $h=0$, the growth rate is zero. For any given $A_{2}$, determining a distribution of solitons, the growth rate increases as the discreteness $h$ is increased. For any given $h$, as $A_{2}$ is increased and $\Delta$ is thereby decreased, the growth rate again increases. The range of $A_{2}$ shown corresponds to between two and eight solitons per wavelength of mode 2 . The growth rate could alternatively be expressed in terms of unscaled time as $1 / t_{e} \propto\left(h^{2} A_{2}\right)^{3} \propto\left(h^{2} / \Delta^{2}\right)^{3}$.

Since $\gamma$ tends to zero in the continuum limit, we conclude that solitons on the periodic $K d V$ model equation are stable with respect to decay to longer wavelengths. Discrete lattice solitons, however, show an exponential decay instability not found in the $K d V$ approximation.

We now consider the cubicly nonlinear periodic lattice, i.e., (1) with $p=2$; we first choose $\alpha>0$, specifically $\alpha=1 / 3$. When the long wavelength mode $A_{2}$ is excited to large amplitude, it is observed to couple to its odd harmonics, until dispersive effects become important. We again see the formation of persistent, free-streaming solitons. This streaming again causes noticeable recurrences in mode 2, although the recurrences are less complete. Further, exponential growth of mode 1 is observed, again dependent on $\mathrm{N}$ and $\mathrm{A}_{2}$.

The cubic instability scaling is, however, somewhat surprising. We find $1 / t_{e} \propto\left(h^{2} A_{2}{ }^{2}\right)^{1.5} \propto\left(h^{2} / \Delta^{2}\right)^{1.5}$, giving $\gamma \propto\left(A_{2}\right)^{3}$ ! The scaled growth rate $\gamma$ is independent of $h$, and therefore does not tend to zero in the continuum limit. This indicates that solutions of the $m K d V$ equation are exponentially unstable.

$m K d V$ Instabilities. We now consider the stability of solutions of the $m K d V$ equation,

$$
u_{\tau} \pm 12 u^{2} u_{\xi}+u_{\xi \xi \xi}=0,
$$

$0 \leqq \xi \leqq L$, with periodic boundary conditions. The two possible choices of sign will be considered explicitly whenever necessary. In order to facilitate the analysis, we consider stationary solutions $u(\xi-b \tau)$; such solutions are obtained by integrating (8) twice. This gives

$$
\begin{aligned}
\frac{1}{2} u_{\xi}^{2} & \pm u^{4}-\frac{1}{2} \mathscr{b} u^{2}-\mathscr{B} u+\mathscr{A} \\
& \equiv \frac{1}{2} u_{\xi}^{2}+\mathscr{P}(u)=0,
\end{aligned}
$$

where $\mathscr{A}$ and $\mathscr{B}$ are constants of integrations. Since the "oscillator polynomial" $\mathscr{P}(u)$ is fourth order, the solution of (9) may be expressed in terms of the Jacobian elliptic functions, e.g., $c n(\xi, q)$, and is called a cnoidal wave. The cnoidal wave is determined by the three parameters $(\mathscr{A}, \mathscr{B}, \mathfrak{b})$, or equivalently by the roots $(a, b, c, d)$ defined by 


$$
\mathscr{P}(u) \equiv \pm(u-a)(u-b)(u-c)(u-d) .
$$

Note that $a+b+c+d=0$, since $\mathscr{P}(u)$ has no cubic term.

Not all $m K d V$ cnoidal waves correspond to possible excitations of the periodic lattice. Since $u \simeq y_{x}$ for small $h$, a periodic wave $u$ will give a periodic lattice displacement $y$ only if the spatial mean of $u$ is zero:

$$
\int_{0}^{L} u d \xi=\int_{0}^{L} y_{x} d x=y(L)-y(0)=0 .
$$

For comparison with the lattice, we consider those cnoidal waves with $\mathscr{B}=0$ which are symmetric in positive and negative $u$; these waves have zero mean. Furthermore, since the system length is arbitrary and may be re-scaled to any value, we consider cnoidal waves with wavelength $\lambda=1$, i.e., fundamental wavenumber $k=2 \pi$. With these two restrictions, the cnoidal waves are specified by a single parameter, which we take to be the cnoidal modulus $q, 0 \leqq q \leqq 1$. For the positive sign choice in (8), the wave is

$$
\begin{aligned}
u(\xi, \tau) & =(8)^{1 / 2} q K \operatorname{cn}[4 K(\xi-b)], \\
b & =(4 K)^{2}\left(2 q^{2}-1\right),
\end{aligned}
$$

and the associated $\mathscr{P}(u)$ has roots $a=i(8)^{1 / 2} K\left(1-q^{2}\right)^{1 / 2}, b=-a$, $c=(8)^{1 / 2} q K, d=-c$. Here, $K(q)$ is the complete elliptic integral of the first kind. For small $q$, the wave is almost sinusoidal; as $q$ approaches 1 , the cnoidal wavetrain becomes a periodic array of the solitons of (7), with alternating positive and negative pulses. For the negative sign choice in (8), the wave is

$$
\begin{aligned}
u(\xi, \tau) & =(8)^{1 / 2} q K \operatorname{sn}[4 K(\xi-\mathscr{C} \tau)], \\
\mathscr{C} & =-(4 K)^{2}\left(1+q^{2}\right),
\end{aligned}
$$

with roots $a=(8)^{1 / 2} K, b=q a, c=-b, d=-a$.

The stability of $m K d V$ cnoidal waves with respect to long wavelength perturbations may be obtained usingWhitham's modulational theory [7], [8]. Our analysis for the $m K d V$ equation parallels Whitham's analysis for the $K d V$ equation. Three equations governing the temporal evolution of slow spatial modulations of the wave parameters are needed; these equations are obtained by averaging conservation equations over a spatial oscillation of the cnoidal wave. The first three $m K d V$ conservation equations [9] are 


$$
\begin{gathered}
\frac{\partial}{\partial \tau}(u)+\frac{\partial}{\partial \xi}\left( \pm 4 u^{3}+u_{\xi \xi}\right)=0, \\
\frac{\partial}{\partial \tau}\left(u^{2}\right)+\frac{\partial}{\partial \xi}\left( \pm 6 u^{4}+2 u u_{\xi \xi}-u_{\xi}^{2}\right)=0, \\
\frac{\partial}{\partial \tau}\left(u^{4} \mp \frac{1}{2} u_{\xi}^{2}\right)+\frac{\partial}{\partial \xi}\left( \pm 8 u^{6}+4 u^{3} u_{\xi \xi}\right. \\
\left.-12 u^{2} u_{\xi}^{2} \mp u_{\xi} u_{\xi \xi \xi} \pm \frac{1}{2} u_{\xi \xi}^{2}\right)=0,
\end{gathered}
$$

where the + or - sign corresponds to that of (8). The averages of quantities appearing in (14) are most easily expressed in terms of the function

$$
\begin{aligned}
W(\mathscr{A}, \mathscr{B}, \mathscr{b}) & \equiv-\oint u_{\xi} d u \\
& =-(2)^{1 / 2} \oint[-\mathscr{P}(u)]^{1 / 2} d u,
\end{aligned}
$$

where the intergal is over one complete cycle of the cnoidal wave. The spatial average of equations (14) may then be expressed as

$$
\begin{aligned}
& \frac{D}{D \tau} \quad W_{\mathscr{A}}=W_{\mathscr{A}} \frac{\partial \mathscr{C}}{\partial \xi}, \\
& \frac{\partial}{D \tau} \quad W_{\mathscr{B}}=W_{\mathscr{A}} \frac{\partial \mathscr{B}}{\partial \xi}, \\
& \frac{D}{D \tau} \quad W_{\mathscr{C}}=W_{\mathscr{A}} \frac{\partial \mathscr{A}}{\partial \xi},
\end{aligned}
$$

where

$$
\frac{D}{D \tau} \equiv \frac{\partial}{\partial \tau}+\mathscr{b} \frac{\partial}{\partial \xi}, W \delta \equiv \frac{\partial W}{\partial \mathscr{A}}
$$

The three coupled nonlinear equations (16) simplify and decouple when expressed in terms of their Riemann invariants. The invariant quantities turn out to be the roots of $\mathscr{P}(u)$ taken two at a time, that is, $a+b, b+c, a+c$. One evolution equation may then be expressed as

$$
\frac{\partial}{\partial \tau}(b+c)+P \frac{\partial}{\partial \xi}(b+c)=0,
$$

where the characteristic speed is 


$$
P=\mathscr{b} \pm \frac{2 W_{\mathscr{A}}(a-b)(a-c)}{w_{\mathscr{A}}+2(d-a)(\partial / \partial d)\left(W_{\mathscr{A}}\right)}
$$

The other two evolution equations are obtained by cyclic permutation of $(a, b, c)$ in (17) and (18).

The stability or instability of the cnoidal wave is determined by whether the roots $(a, b, c, d)$ are real or complex. When all four roots are real, the characteristic speeds are real, and small perturbations oscillate rather than grow: e.g., $b+c=b_{0}+c_{0}+(\delta b+\delta c)$ cos $[\kappa(\xi-P \tau)]$. When two roots are real $(c>d)$ and two are complex $\left(a=b^{*}\right)$, two characteristic speeds are complex, and small perturbations grow exponentially: e.g., $\quad b+c=b_{0}+c_{o}+1 / 2(\delta b+\delta c)$ $\{\exp [i \kappa(\xi-P \tau)]+\exp [-i \kappa(\xi-P \tau)]\}$. Note that at least two roots must be real to define a bounded cnoidal wave.

For the $m K d V$ equation (8) with negative sign choice, all cnoidal waves are stable: the polynomials $\mathscr{P}(u)$ associated with bounded waves all have four real roots. For the $m K d V$ equation (8) with positive sign choice, some cnoidal waves are unstable, corresponding to polynomials $\mathscr{P}(u)$ with two real and two complex roots. Specifically, the $\mathscr{B}=0$, zero mean cnoidal waves of (12) are unstable for this sign choice; from (17) and (18), a sinusoidal modulation with wavenumber $\kappa$ will have exponential growth rate

$$
\begin{aligned}
\gamma=\operatorname{Im}(P) \kappa & \\
& =\frac{32 q\left(1-q^{2}\right)^{1 / 2} K^{3}\left[q^{2} E+\left(1-q^{2}\right)(K-E)\right]}{q^{2} E^{2}+\left(1-q^{2}\right)(K-E)^{2}} \kappa .
\end{aligned}
$$

Here, $K(q)$ and $E(q)$ are the complete elliptic integrals of the first and second kinds.

The growth rate of (19) is valid only for slow spatial modulations, i.e., $\kappa / k \ll 1$. Stability predictions for shorter wavelength perturbations may be obtained by numerically solving the linearized stability equation. We consider $u(\xi, \tau)=u_{0}(\xi-b \tau)+v(\xi-b \tau) \exp (i \nu \tau)$, where $u_{o}$ is the cnoidal wave of (12). This gives the linearized perturbation equation

$$
i v v-b v_{\xi}+12\left(u_{0}^{2} v\right)_{\xi}+v_{\xi \xi \xi}=0 .
$$

We solve (20) in Fourier space, keeping only a finite number of modes. The linear operator on $v$ is then represented by a matrix, determined by $b$ and the Fourier modes of $u_{0}$. We find the eigenvalues $\nu$ and eigenvectors $v$ of this matrix using the numerical routines of EISPACK [10]. The Fourier modes of $v$ are separated from the modes of $u_{0}$ by 
the wavenumber $\kappa$. The numerical eigenvalue results agree with the analytic results, for small $\kappa / k$. We find, however, that shorter wavelength modulations tend to be stabilized. In Figure 3 we summarize the in-

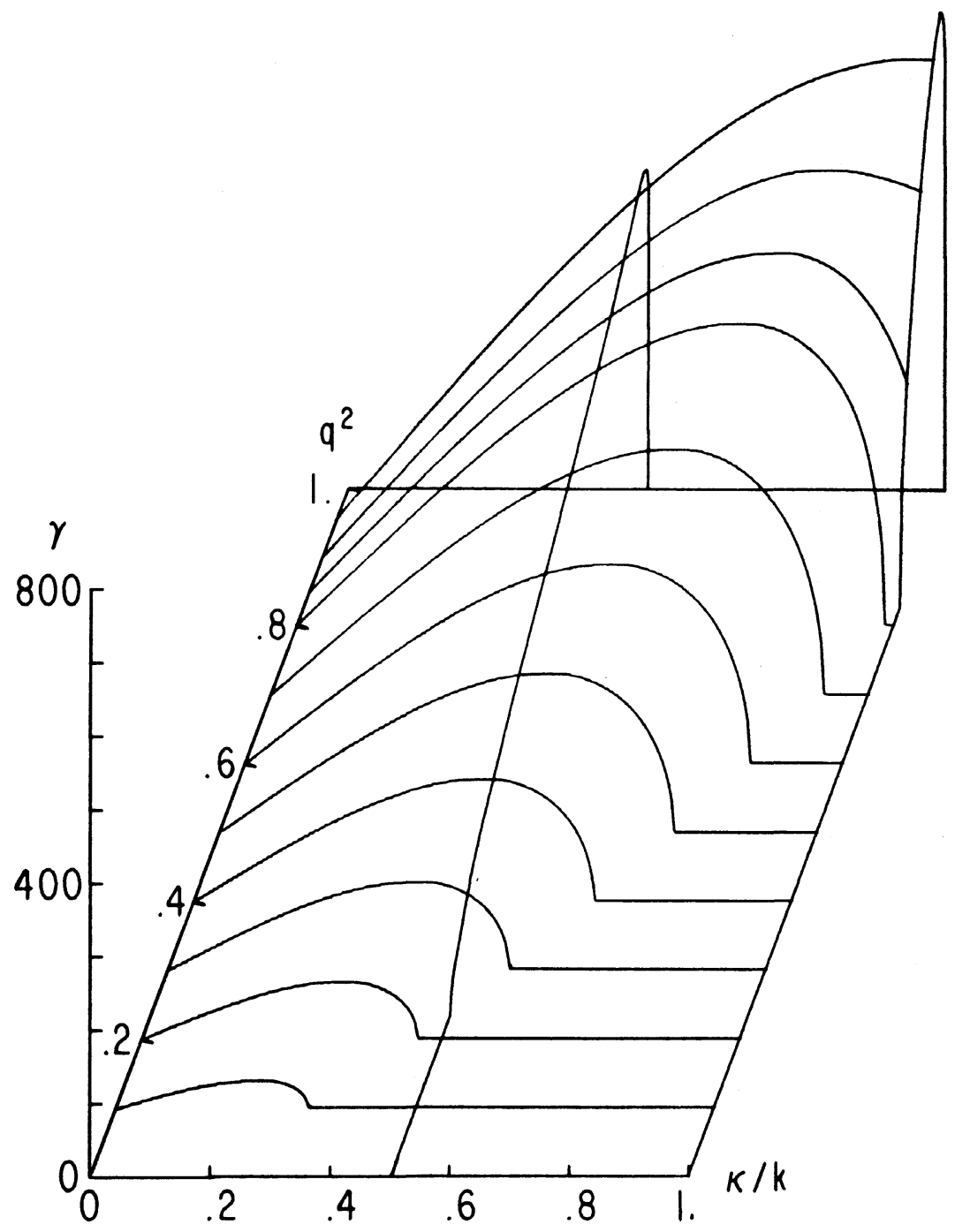

Figure 3. $\mathrm{mKdV}$ cnoidal wave instability rate $\gamma$ vs scaled perturbation wavenumber $\kappa / k$ and wave modulus $q^{2}$. 
stability results for the $\mathscr{B}=0$, zero mean cnoidal waves: here, we display the growth rate $\gamma=\operatorname{Im}(\nu)$ vs $\kappa / k$ and $q^{2}$, with two cross-sections at $\kappa / k=1 / 2$ and 1 .

Some insight may be gained into the $\kappa / k=1$ instability by analysis of (20) with $u_{0}$ given by (12). The eigenvalue $\nu$ is purely imaginary, and goes to zero at two limits of $q$. The eigenvector at the lower limit may be seen to be $v=c n^{2}(4 K \xi, q)-\left(2 q^{2}-1\right) / 2 q^{2} \propto u_{0}{ }^{2}-\left\langle u_{0}^{2}\right\rangle$; and the limit is determined by $K(q)=2 E(q)$, giving $q=(.826)^{1 / 2}$. The eigenvector at the upper limit of $q=1$ may be seen to be $\operatorname{sn}(4 K \xi, q) \operatorname{cn}(4 K \xi, q)$. This latter eigenvector approximates the spatial derivative of $u_{0}$, differing by a factor of \pm 1 , with additional corrections of order $\left(1-q^{2}\right)^{1 / 2}$. It corresponds to shifting the positive pulse in one direction, and the negative pulse in the other direction; that is, the two pulses are moving closer together exponentially with time.

Cubicly Nonlinear Lattice Instability. We are able to obtain quantitative agreement with the $m K d V$ predictions, by exciting cnoidal waves on the cubicly nonlinear lattice and measuring instability rates. A zero mean cnoidal wave $u(\xi)$ can be excited on the lattice by constructing displacement and velocity functions $y$ and $y_{t}$ which correspond to $u(\xi)$ through (4). The functions $y$ and $y_{t}$ are constructed from the positively travelling modes $A_{m}$. We also consider the case where identical waves are excited in each direction, i.e., $B_{m}=A_{m}$. For large $N$, the mass displacements and velocities $y_{j}$ and $y_{j t}$ will accurately represent the cnoidal wave; for small $N$, the representation will be more approximate.

Lattice instability results for the positive sign choice in (1) are shown in Figure 4. In Figure 4a we excite cnoidal waves with $\lambda=1$ (parametrized by $q$ ) on the lattice with length $L=2$; that is, the fundamental mode of the cnoidal wave is $A_{2}$. We observe exponential growth from noise of the lattice mode $A_{1}$. This corresponds to the $m K d V$ modulational instability with $\kappa / k=1 / 2$, shown as a cross-section in Figure 3 and as the solid curve in Figure 4a. The data points are the scaled instability rates $\gamma=1 / \tau_{e}$, for various $N$. The hollow symbols are for waves excited in one direction only, while the solid symbols are for waves excited in both directions. The uncertainty in the determination of $\gamma$ is about $\pm 5 \%$. The lattice growth rates are seen to asymptotically approach the $m K d V$ prediction, as $N$ becomes large.

In Figure $4 \mathrm{~b}$, we excite cnoidal waves with $\lambda=1$ on the lattice with periodicity $L=1$; that is, the fundamental mode of the cnoidal wave is $A_{1}$. The initial cnoidal wave contains only the odd harmonics $A_{3}, A_{5}$, etc., but the "forbidden modes" $A_{2}, A_{4}$, etc. are observed to grow from noise. We measure the exponential growth of $A_{2}$ and plot the scaled 

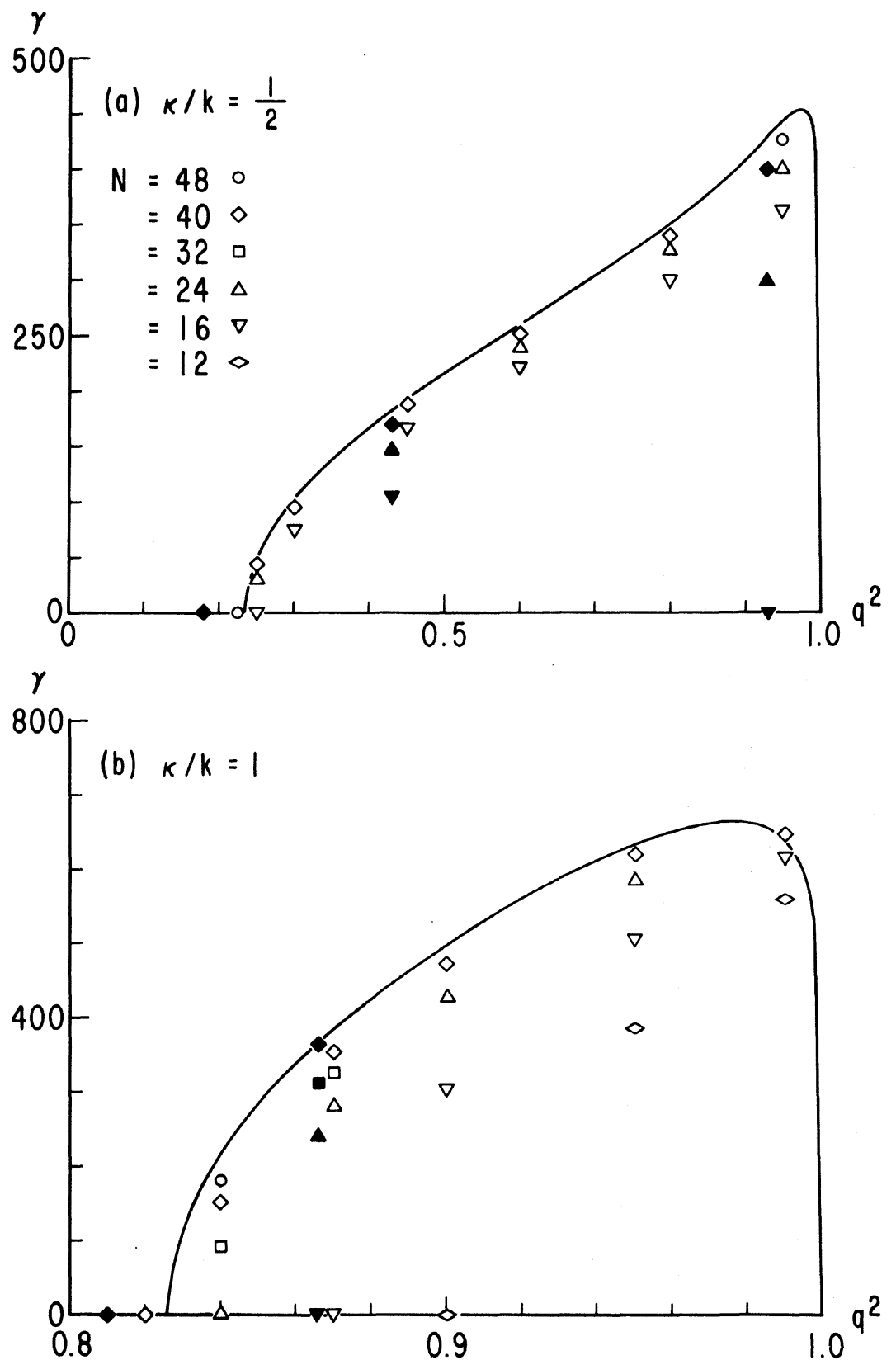

Figure 4. Scaled lattice instability rate $\gamma$ (data points) and $m K d V$ prediction (solid curves) for cnoidal waves with modulus $q^{2}$. (a) Growth of longer wavelength mode. (b) Growth of "forbidden modes." 
growth rate $\gamma$. Again, the observed lattice instability corresponds to that predicted by the $m K d V$ equation. The $q \lesssim 1$ data for waves travelling in both directions is erratic, however, and is not shown. It is not yet known whether this is due to insufficiently large $N$, or to a new instability mechanism.

The cubicly nonlinear lattice instability is interesting in that there is a definite, analytically accessible cut-off: the $\kappa / k=1$ instability is quenched for $q^{2}<.826$, as is the $\kappa / k=1 / 2$ instability for $q^{2}<.235$. This may be viewed as due to the boundary conditions imposed: any given cnoidal wave (modulus $q, \lambda=1$ ) will become unstable as the periodic system is made "longer", that is, as longer wavelength perturbations are allowed. The data also shows that the waves may become stable as $N$ is decreased, but this limit is not contained in the continuum $m K d V$ approximation.

For the cubicly nonlinear lattice with $\alpha<0$ in (1), we observe no similar instability. This corresponds to the stability of cnoidal wave solutions of the $m K d V$ equation with negative sign choice. This lattice is, however, subject to "explosive dissociation" (as is the $p=1$ lattice), since for large displacements the potential energy may become infinitely negative.

The instability of cnoidal waves on the cubicly nonlinear lattice provides the explanation for the instability observed with sinusoidal initial conditions. The large amplitude wave forms a series of solitons of various amplitudes. Since the basic configuration is changing with time as the solitons stream through one another, an exact stability analysis would be difficult. However, the scaled growth rate $\gamma$ is the same order of magnitude as that observed for cnoidal waves of similar amplitude and wavelength.

Conclusions and Future Directions. We have seen that lattice solitons may be persistent, and dominate the dynamics, yet still show instabilities. For the quadratically nonlinear lattice, the instability scales with terms neglected in the $K d V$ approximation, and thus tends to zero in the continuum limit. For the cubicly nonlinear lattice, the instability is only seen for one sign of the nonlinearity; when present, however, it is seen even in the continuum limit, and is in fact well modeled by the $m K d V$ equation.

Solitons have been observed for many different nonlinearities. Yet it is still an open question, What classes of nonlinearities give rise to the various types of instabilities? A first step in this direction would be to understand the periodic $m K d V$ instability within the framework of the Inverse Scattering Method, which has been applied to many nonlinear evolution equations [3]. Comparison can be made with singular systems 
such as the Toda lattice which shows no instabilities and is known to be integrable [11]. Exponential instability is the signature of "stochastic" behavior, and leads to loss of information about the previous state of the system [12]; but what fraction of phase space do these instabilities cover? How does one characterize the soliton state when these instabilities saturate? Lattice solitons have been conjectured to contribute significantly to the thermal conductivity of crystals [13]. Can instabilities determine an effective "lifetime" for a soliton trajectory, thereby associating an energy gradient with a heat flow? The answer to these and other questions may yet help establish. nonlinear dynamical effects as the foundation of present statistical theories.

\section{REFERENCES}

1. E. Fermi, J. Pasta, and S. Ulam, Studies of nonlinear problems. I., Los Alamos Report LA-1940 (1955); reprinted in Nonlinear Wave Motion, A. C. Newell, ed., Lectures in Applied Math., Vol. 15, AMS, Providence, R.I., 1974, pp. 143-156.

2. N. J. Zabusky and M. D. Kruskal, Interaction of "solitons" in a collisionless plasma and the recurrence of initial states, Phys. Rev. Lett. 15 (1965), 240-243; N. J. Zabusky, A Synergetic approach to problems of nonlinear dispersive wave propagation and interaction, Nonlinear Partial Differential Equations, W. F. Ames, ed., Academic Press, New York, 1967, pp. 223-258.

3. A. C. Scott, F. Y. F. Chu, and D. W. McLaughlin, The soliton: A new concept in applied science, Proc. IEEE 61 (1973), 1443-1483.

4. J. Ford, The transition from analytic dynamics to statistical mechanics, Adv. Chem. Phys. 24 (1973), 155-185.

5. B. V. Chirikov, F. M. Izrailev, and V. A. Tayursky, Numerical experiments on the statistical behavior of dynamical systems with a few degrees of freedom, Comput. Phys. Commun. 5 (1973), 11-16.

6. R. L. Bivins, N. Metropolis, and J. R. Pasta, Nonlinear coupled oscillators: Modal equation approach, J. Comput. Phys. 12 (1973), 65-87.

7. G. B. Whitham, Linear and Nonlinear Waves, Sec. 16.1, Wiley, New York, 1974.

8. C. F. Driscoll and T. M. O'Neil, Modulational instability of cnoidal wave solutions of the modified Korteweg-deVries equation, J. Math. Phys. 17 (1976), 1196-1200.

9. R. M. Miura, C. S. Gardner, and M. D. Kruskal. Korteweg-deVries equation and generalizations, II. Existence of conservation laws and constants of motion, J. Math. Phys. 9 (1968), 1204-1209.

10. B. T. Smith, et al., Matrix Eigensystem Routines-EISPACK Guide, 2nd Edition, Lecture Notes in Computer Science, Vol. 6, Springer-Verlag, New York, 1976.

11. M. Hénon, Integrals of the Toda lattice, Phys. Rev. B 9 (1974), 1921-1923.

12. D. V. Anosov and Ya. G. Sinai, Some smooth ergodic systems, Russ. Math. Surveys 22, No. 5 (1967), 103-167.

13. N. J. Zabusky, Solitons and energy transport in nonlinear lattices, Comput. Phys. Commun. 5 (1973), 1-10.

University of California at San Diego, la Jolla, CA 92093 
